\title{
CHARACTERIZATION AND GROUPING OF PHAGE TYPES OF SALMONELLA PARATYPHI B, ESPECIALLY OF A NEW TYPE 'SITTARD', BY SENSITIVITY TO TYPE PHAGES AND BY LYSOGENIC PROPERTIES
}

\author{
By R. TH. SCHOLTENS \\ National Institute of Public Health, Utrecht
}

\section{INTRODUCTION}

One important scientific discovery leads to others, which are often made simultaneously by different authors. In this way, the discovery of the Vi antigen by Felix \& Pitt (1934) was followed by the finding that this antigen plays a part in the host-cell relationship between typhoid bacilli and certain phages (Craigie \& Brandon, 1936; Sertic \& Boulgakov, 1936; Scholtens, 1936); and the subdivision of Salmonella typhi by means of specifically adapted preparations of Vi phage type II, discovered by Craigie \& Yen (1938), was followed by the observation by Boyd (1950) that a type classification of Salm. typhi-murium can be based on the identity of the phages produced spontaneously by different strains. Scholtens (1950) demonstrated a partial relationship between the types of Salm. paratyphi $B$ in the Felix \& Callow system and the characteristics of the phages they produce spontaneously, the properties of which can be used, in conjunction with and complementary to sensitivity to a set of phages, to characterize types of this species. Similar investigations were made at the same time at the Pasteur Institute by Nicolle, Hamon \& Edlinger (1951), Hamon \& Nicolle (1951) and Nicolle \& Hamon (1951). Felix \& Callow also reported similar results (1951).

Scholtens $(1952 a, b)$ showed that if different Salm. paratyphi $B$ types are grown for a sufficient time in mixed culture with a certain strain called 'Midwoud', mixtures of phage appear which are not demonstrable in pure cultures of the same strains.

In the present paper, the phage types of Salm. paratyphi $B$ are shown to fall into groups such that each member of a group when grown in mixed culture with 'Midwoud' produces characteristic phages. Moreover, the individual strains comprising such groups, when grown in pure culture, produce phages which are related to one another.

A new type, called 'Sittard', difficult to place in the system of Felix \& Callow, is characterized by its lysogenic properties and its sensitivity to phages. Like type 'Midwoud', in mixed culture with other types it gives rise to characteristic phage mixtures.

A few strains from sporadic cases are described which may, on similar grounds, prove to be new types. 
(1) Host range

METHODS

Phage preparations were titrated by the method of Craigie \& Yen to determine the range of hosts sensitive to them. The critical test dilution, when found, was tested on several strains.

\section{(2) Serological reactions}

$0.3 \mathrm{ml}$. of a phage preparation at critical test dilution was added to an equal volume of each of a series of twofold dilutions, from $1 / 50$ to $1 / 12,800$, of the sera to be examined. After $1 \mathrm{hr}$. in a water-bath at $50^{\circ} \mathrm{C}$. and a further $18 \mathrm{hr}$. at room temperature, a drop of each mixture was placed on an agar surface inoculated with the sensitive strain. Readings were made after $24 \mathrm{hr}$. at $37^{\circ} \mathrm{C}$.

The phages belonging to antigenic groups I, II, IV, VI and VII (Table 1) show hardly any cross-reactions. Group II antisera also neutralize the typing phage 'Dundee' of the Felix \& Callow system. It must be emphasized here that this does not mean a serological identity. The serology of the phages is probably complex, and more refined methods of examination analogous to a receptor analysis of bacteria as introduced by Weil \& Felix (1920) could perhaps show differences.

\section{(3) Detection of lysogenicity}

Lysogenicity has been demonstrated by two different methods, which will be called 'the direct method' and 'the mixed culture method'.

(a) The direct method. This method is applied to pure cultures of unmixed type. A broth culture of the strain under examination is filtered and a drop of the filtrate is placed on an agar surface inoculated with a sensitive strain. After $24 \mathrm{hr}$. incubation at $37^{\circ} \mathrm{C}$. plaques appear. By touching a plaque under the colony microscope with the point of a pipette, phage and sensitive bacteria are picked into broth and incubated at $37^{\circ} \mathrm{C}$. for $24 \mathrm{hr}$. This broth culture is filtered or shaken with chloroform.

An alternative method is to grow the strain under investigation in mixed culture with a sensitive indicator strain for a short time, say $24 \mathrm{hr}$.

(b) The mixed culture method. Sixty $\mathrm{ml}$. of broth is inoculated with a drop of a fresh broth culture of each of two strains belonging to different types. After 5-10 days' incubation at $37^{\circ} \mathrm{C}$. phages are isolated from the mixed culture as described above for the direct method.

The author saw this method applied in the laboratory of Flu (1933) to Vibrio cholerae and Shigella flexneri. Lisbonne \& Carrère discussed the production of bacteriophage by bacterial antagonism as long ago as 1922. As stated above Scholtens $(1950,1952 a, b)$ found that strains of different types of Salm. paratyphi B grown in mixed culture with strains of several other types and given a long incubation yielded phages not found in pure cultures of the same strains by the direct method.

The characteristics of the phages found by the mixed culture method depend on the type of each of the strains in the mixture. Certain types possess to a high degree the power to produce phages in mixed cultures. One such type is type 'Midwoud', 
strains of which react like strains of type 'Beccles' in the Felix \& Callow system but can be differentiated from 'Beccles' by their lack of sensitivity to the phage from type 'Dundee' of this system. It will be shown that two groups of types exist which yield by the mixed culture method with type 'Midwoud' mixtures of phages different in host range but parallel in serological behaviour.

\section{(4) Examination of phage preparations for purity and constancy of character}

With a suitable dilution of the phage preparation, separate plaques were obtained. Phages were subcultured from fifteen plaques and examined. Three passages were made on the indicator strain with which the phage was isolated and the examination was repeated. Only phages which could be shown to yield identical subcultures (or, in some cases, regularly the same variants) were considered to be pure and of constant character.

\section{(5) Examination of thermostability}

One $\mathrm{ml}$. of a suitable dilution of the phage preparation was placed in a culture tube and heated for half an hour in a water-bath. A drop was placed on an agar plate inoculated with a sensitive strain. After $24 \mathrm{hr}$. incubation the result was compared with that of an unheated control which showed about 200 plaques.

Phages found in mixed cultures of strains of types $3 b, 22$ and 'Dundee' with strains of type 'Midwoud'

Mixed cultures of type 'Dundee' with type 'Midwoud' yield a mixture of phages, one of which attacks all types of Salm. paratyphi B except types 'B.A.O.R.' and 'Midwoud', while another attacks all types except $3 b$ and 'Dundee' (Scholtens, $1952 a, b)$ and a newly defined type, ' 22 ', classified as type 'Beccles' in the Felix $\&$ Callow system, and itself carrying a phage of Scholtens' group $I$.

Strains of $3 b, 22$ and 'Dundee' in mixed cultures with strains of 'Midwoud' produced, after prolonged incubation, phages which attacked all types except $3 b$, 'Dundee' and 22, and also other phages which attacked all types except 'B.A.O.R.' and 'Midwoud'. No other type was found which produced this mixture of phages.

Serologically, strains of $3 b$ and 22 yield, examined by the direct method, no group VI phage; type 'Dundee' yields phages of group I and VI. Now, all the phages from the mixtures ' $3 b /$ Midwoud' and '22/Midwoud' belonged to group II. The serology of the phages from the mixture 'Dundee/Midwoud' was more complicated; two serological forms were found, one belonging to group II and the other reacting with group II sera to low titre and with group VI sera to high titre.

In phages from mixtures 'Dundee/Midwoud' serological group is independent of host range, so that four different phages are found (Scholtens, 1952 $a, b$ ).

\section{Phages found in mixed cultures of type 'Midwoud' with other types}

Mixed cultures of 'Midwoud' with type I of Felix \& Callow, or 'Beccles' (variety 'Meppel', Scholtens, 1952) or 'Jersey' or 'Sittard' (see below) regularly yielded phage which attacked all types except 'B.A.O.R.' and 'Midwoud', and also one 
which attacked 'B.A.O.R.' and 'Midwoud' in critical test dilution and had a weaker action on the other types (Table 1 ).

Strains of types $3 a$ (e.g. strain B 62) and $3 a$ I (e.g. strain B 1305, variety 'Schiedam' of Scholtens) sometimes produced similar phages in mixed culture with 'Midwoud', but they frequently failed to do so. Some of these types produced serological group I phage by the direct method, but none of them produced group VI phage. Serologically, all phages found in mixed cultures with 'Midwoud' belonged to group II.

Eight different strains of 'Midwoud' were grown in mixed culture with strains which, like 'Dundee', produced by the direct method phages of groups I and VI. One of these, Pty 14390, resembling strain $3 a$ I B 624 of Felix and Callow in its phage sensitivity, when mixed with one of the eight 'Midwoud' strains, produced in one experiment phages not yielded by either of the two strains by the direct method. In further experiments with this strain and with other strains of type $3 a I$ (Leeuwarden) the phenomenon could not be repeated. The phages obtained on this occasion had the same host ranges as the two phages from the mixture 'I Felix \& Callow/Midwoud' (Table 1); serologically one form belonged to group II and the other reacted with sera of both groups II and VI. Both serological forms were found associated with each host range.

A further strain, 14487, in mixed culture with each of the eight 'Midwoud' strains regularly produced phages not found by the direct method in any of the strains. Unlike type $3 a I$ ('Leeuwarden') this strain was sensitive to the phages found in the culture mixtures ' $22 /$ Sittard' and ' $3 b /$ Sittard' and must therefore be designated as a separate type, '14487'. After a longer incubation in mixed culture with 'Midwoud', 14487 regularly produced a mixture of phages, resembling in host range the phages found in the mixture 'type I Felix \& Callow/Midwoud'.

Serologically, the phages were more complicated: one serological form was inhibited by group II sera only, and another by group VI sera. Both serological forms were found associated with each host range.

Table 2 summarizes the properties of phages obtained by growing other types in mixed culture with type 'Midwoud'. It will be seen that $3 b, 22$ and 'Dundee' form one group, and type I of Felix \& Callow, 'Beccles' (Meppel), and 'Jersey' another with which $3 a, 3 a I$ (B 1305) and $3 a I$ (B624 or 14390) and 'Sittard' and 14487 are associated. Whether this grouping of types can be reliably subdivided is a question for further research. Further research will also be needed to show whether this grouping is related to that described by Nicolle et al. (1951), Hamon \& Nicolle (1951) and Nicolle \& Hamon (1951). These workers distinguished between latent phages that are 'type-determinant' and those that are 'indifferent' (i.e. non-typedeterminant), and they correlated their findings with those of Felix \& Callow (1951) on 'natural' and 'adapted' phages. The International Committee for Enteric Phage Typing at its meeting during the Sixth Congress for Microbiology in Rome, 1953, initiated a co-ordinated investigation of this problem on an international basis (Report of Proceedings, 1953). 


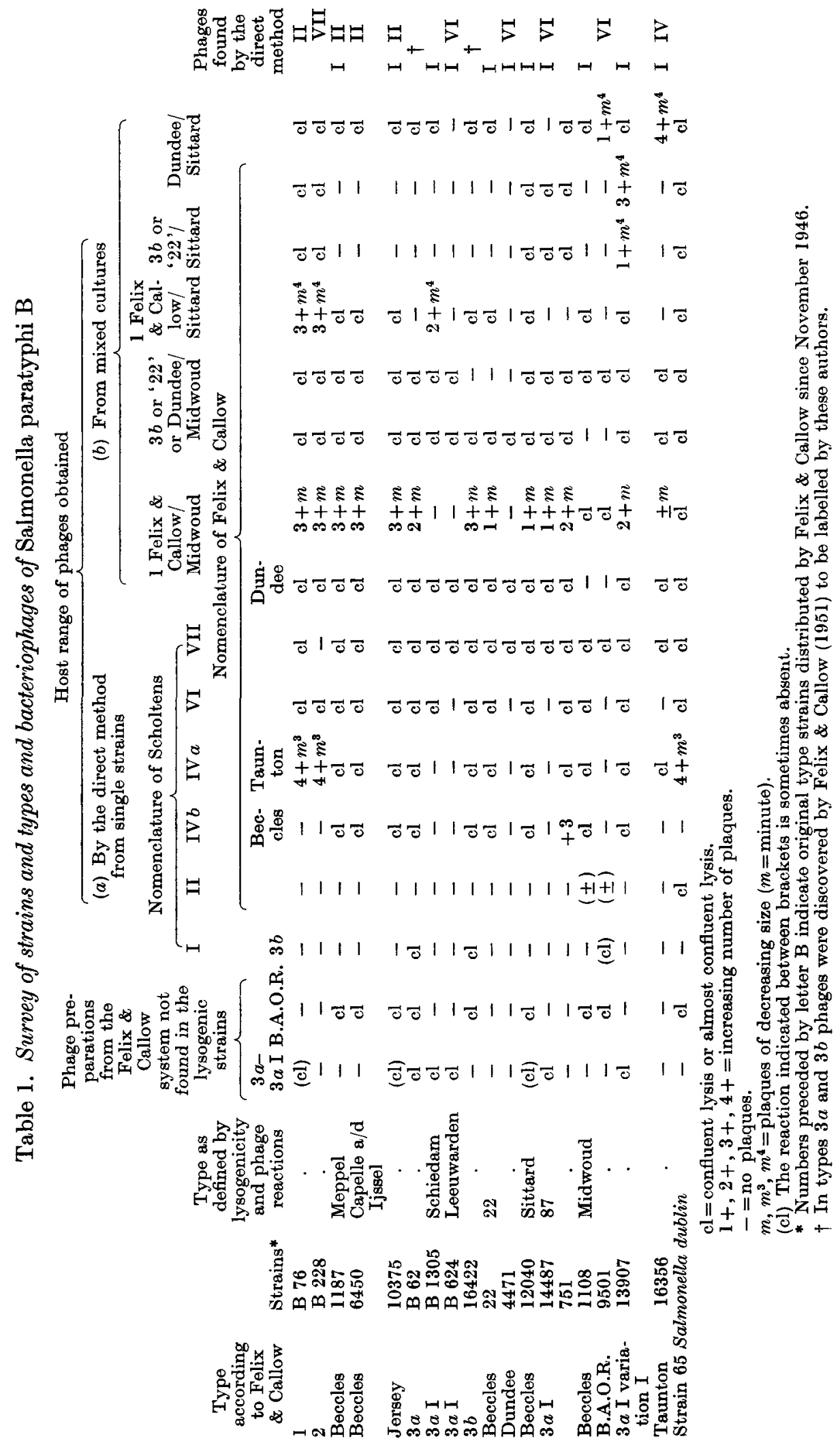




\section{Identification of the new type, 'Sittard'}

Another type was found which resembled type 'Midwoud' in that, when grown in mixed culture with strains of various other types, it gave rise to phages not to be found by the direct method in the pure strains.

In 1948 there occurred an outbreak of paratyphoid fever traced to steamed herring from a fish smoking factory in the village of Lemmer. When typed according to the Felix \& Callow system some of the strains from this outbreak showed the reactions of type $3 a \mathrm{I}$ variation $\mathrm{I}$ and some of type 'Beccles'. The amount of lysis given by phages $3 a$ and $3 a \mathrm{I}$, which differentiate between types $3 a \mathrm{I}$ variation 1 and 'Beccles', varied between 'absent' or 'hardly visible' and

Table 2. Phages produced in mixed cultures of various types with type Midwoud

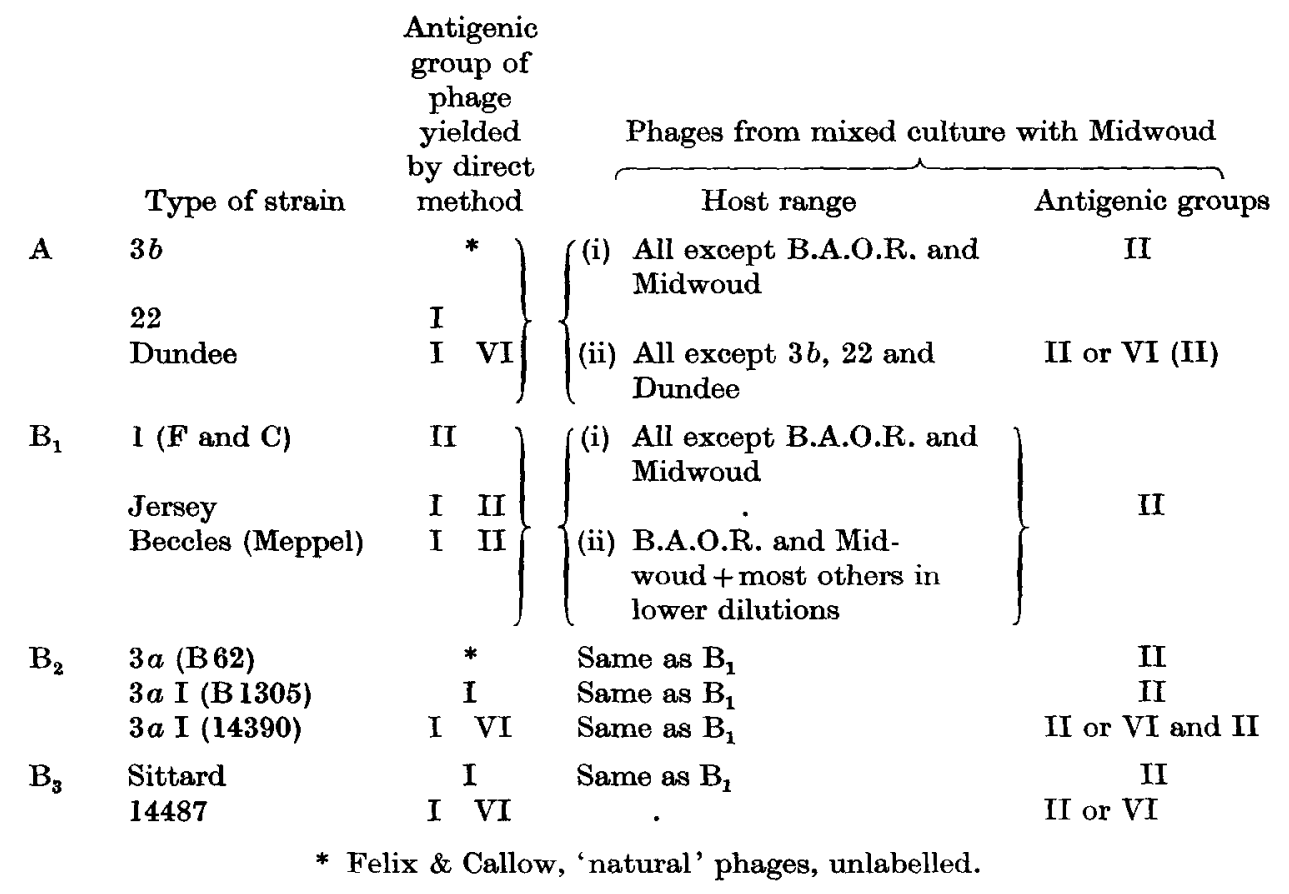

'conspicuous'. Patients who first excreted strains typed as 'Beccles' produced a few weeks later strains which were typed as $3 a \mathrm{I}$ variation 1 . A single strain gave on one day the reaction of type $3 a \mathrm{I}$ and on the next day of 'Beccles'. Some strains were sent to the Central Enteric Reference Laboratory in London, where all were found by Dr Felix to be of type 'Beccles'. The same strains were sent to Paris, to Dr Nicolle, who found them to be of type $3 a \mathrm{I}$. Various attempts to adapt the type 1 phage of Felix \& Callow to the Lemmer strains failed; which is not surprising, for only three specific adaptations of this phage have been reported in the 10 years during which the Felix \& Callow system has been used.

A study of the lysogenic properties of the Lemmer strains, described below, showed that they constitute a new type, called 'Sittard'. 


\section{The lysogenic properties of strains of type 'Sittard'}

Strains of type 'Sittard' carry phage I ('natural' phage $3 b$ of Felix \& Callow). No other phage was found in these strains although filtrates of their cultures were tested on all the phage types of Salm. paratyphi $B$, and nearly all the type strains of salmonellas in the Kauffmann-White scheme (obtained from the Salmonella Centre, Holland), and with the type strains for Salm. typhi-murium of Boyd.

Prolonged incubation of strains of type 'Sittard' in mixed culture with strains of other types yielded phages other than those obtained from the individual strains by the direct method. Strains of types $3 b, 22$ and 'Dundee' when grown in mixed culture with 'Sittard' yielded phages which were similar in many respects.

In the mixtures ' $3 b /$ Sittard' and '22/Sittard' a phage was found that attacked, besides the 'Sittard' strains, types 1 and 2 of the Felix \& Callow system and some other strains belonging presumably to new types, such as 14487 , to be described below (Table 1). Serologically, this phage belonged to group II. As this phage does not attack any subtypes of type 'Beccles' of the Felix \& Callow system, it can be used to differentiate 'Sittard' from 'Beccles'.

On strains of type $3 a \mathrm{I}$, variations 1 and 2 of Felix \& Callow, this phage may produce plaques so small as to be hardly visible with the aid of a hand-lens, an effect sufficiently different from its action on strains of type 'Sittard' to allow its use for the differentiation of 'Sittard' from $3 a I$ variations 1 and 2 .

In the mixed culture 'Dundee/Sittard' a serologically more complex mixture of phages was generated than in the mixed cultures ' $3 b$ or $22 /$ Sittard'. A first phage was similar to that found in the mixed cultures ' $3 b /$ Sittard' and '22/Sittard', except that its effect on type $3 a \mathrm{I}$, variations 1 and 2 was slightly more conspicuous. A second phage corresponded in serology and heat resistance to phage VI, produced by the direct method from type 'Dundee', but differed from phage VI in plaque form and in virulence. This phage which produces plaques with slightly turbid bases also produces a few plaques with clearer bases when strong preparations are titrated. At its critical test dilution, and also in stronger concentrations, it attacks the types also attacked by phage VI, and, used in stronger concentration, it moreover attacks types 'Taunton' and 'B.A.O.R.', which are not attacked by phage VI itself. Preparations of this phage often show a higher titre, e.g. 10-4, than do preparations of phage VI, which are rarely stronger than $10^{-2}$.

Two other phages in the mixture were neutralized to high titre by group VI antisera as well as by group II antisera. (Table 3). One of these phages attacked strains of types 1, 2 and 'Sittard' and some further strains, the other phage attacking the same strains as phage VI, some of them showing smaller plaques.

Finally, phages showing a low heat resistance (cf. mixtures ' 1 Felix \& Callow/Sittard', described below) were found, but rarely.

These phages resembled phage VI antigenically, but were rapidly destroyed at $58^{\circ} \mathrm{C}$., whereas phage VI itself is stable at $60^{\circ} \mathrm{C}$., though rapidly destroyed at $65^{\circ} \mathrm{C}$., and phages reacting with sera of group II are more resistant at $65^{\circ} \mathrm{C}$. and are rapidly destroyed at $70^{\circ} \mathrm{C}$. Two different host ranges were represented.

It is clear, therefore, that types $3 b, 22$ and 'Dundee' show a special behaviour 
when mixed with type 'Sittard', just as they do when mixed with type 'Midwoud'. In both cases, mixtures containing types $3 b$ or 22 produce phages belonging to group II, while 'Dundee' produces a complex mixture of phages.

\section{Phages found in mixed cultures of type 1 of Felix \& Callow with 'Sittard'}

Filtrates of strains of type 1 of Felix \& Callow do not lyse 'Sittard'. If, however, a strain of type 'Sittard' is incubated in mixed culture with one of type 1 of Felix $\&$ Callow, or if it is incubated for the same length of time in broth to which a few $\mathrm{ml}$. of a filtrate of a broth culture of type 1 has been added, a phage is produced.

The host range of this phage is shown in Table 1. It was not neutralized by antisera to phages of groups I, II, IV or VI, but showed considerable cross-reaction with antisera to group VII. An antiserum prepared against it, however, did not neutralize phage VII.

\section{Table 3. Neutralization of phages from Dundee/Sittard mixed cultures} by specific antisera

\begin{tabular}{|c|c|c|c|c|c|c|c|c|c|c|c|}
\hline \multirow[b]{2}{*}{ Phages } & \multicolumn{5}{|c|}{ Serum 27, anti-phage VI } & \multicolumn{5}{|c|}{$\begin{array}{l}\text { Serum 252, } \\
\text { anti-phage } 23 \text { Dundee/Sittard }\end{array}$} & \multirow[b]{2}{*}{ Control } \\
\hline & $1 / 50$ & $1 / 200$ & $1 / 800$ & $1 / 3200$ & $1 / 12,800$ & $1 / 50$ & $1 / 200$ & $1 / 800$ & $1 / 3200$ & $1 / 12,800$ & \\
\hline VI & - & - & - & - & +4 & +4 & +4 & +4 & +4 & +4 & +4 \\
\hline 66.3 .1 & - & - & - & - & \pm & - & - & +3 & +3 & +3 & +3 \\
\hline 1.2 .8 & - & - & - & - & +3 & - & - & - & +3 & +4 & +4 \\
\hline 23 & +4 & +4 & +4 & +4 & +4 & - & - & - & - & +2 & +4 \\
\hline
\end{tabular}

Phage VI $=$ phage produced in pure cultures of strains of types Dundee, $3 a I$, e.a. 66.3.1, 1.2.8, 23= phages from the mixed culture Dundee/Sittard.

$\pm,+3,+4=$ increasing number of plaques.

$-=$ no plaques.

This phage showed a remarkably low heat resistance. A preparation which, unheated, gave over 200 plaques, produced none after heating at $56^{\circ} \mathrm{C}$. for $30 \mathrm{~min}$.

In the same way, new phages were found in the mixed cultures of 'Sittard' and 'Jersey'; but in mixtures with types $3 a$ and $3 a \mathrm{I}$ (subtype 'Schiedam', Scholtens, $1950,1952 a, b)$ and with subtype 'Meppel' of type 'Beccles' (Scholtens, 1952a, $b$ ) the only phages found so far were those obtainable from the individual strains.

\section{Further justification of the designation of the type 'Sittard'}

It has been stated above that strains from the Lemmer outbreak have been designated as a new type because, although they proved to be of type 'Beccles' or $3 a \mathrm{I}$ variation 1 on the Felix \& Callow system, they differ from these types in being sensitive to a phage found in culture mixtures ' $3 b /$ Sittard' and '22/Sittard' (Table 1). If the biological basis of the new type is sound, then other strains showing sensitivity to the same range of phages might be expected to show the same lysogenic properties. To test this point, each of seven strains of type 'Sittard' was grown in mixed culture with each of five strains of type 1 of Felix \& Callow.

After a week the mixed cultures were examined for their phage content. In Table 4 the result with seven of the 'Sittard' strains is shown and the constancy of lysogenic properties can be judged. The result as shown is partly a function of 
time of incubation, for after another week of incubation a few more of the mixed cultures had produced phage.

The characteristic phage already described as occurring in the mixed culture ' 1 Felix \& Callow/Sittard' was not produced in all mixed cultures; however, at least one strain of type 'Sittard' produced it in mixed culture with all five type 1 strains. Table 4 also shows that the strain of type 1 called 780 was more apt to produce the phage in mixed cultures than were the other type 1 strains.

The seventh strain showing the sensitivity range of 'Sittard', 47, failed to produce the characteristic phage in mixed cultures, and was included in a further experiment, wherein another ten 'Sittard' strains were grown in mixed culture with strain 780 .

In this experiment, strain 47 was the only one which did not produce the characteristic phage with 780 : but, in mixed culture with strain 22,47 and ten further 'Sittard' strains all produced the group II phage which always appeared in mixed cultures of 22 with 'Sittard'.

Table 4. Frequency of appearance of phages in mixed cultures of strains of types ' 1 Felix \& Callow/Sittard' after 5 days' incubation

No. of strains of type Sittard

10,648
12,040
7,651
10,807
6,579
13,650

47

\begin{tabular}{ccccc}
\multicolumn{5}{c}{ No. of strains of type I Felix \& Callow } \\
780 & 6803 & 2451 & 11374 & B 76 \\
+ & + & + & + & + \\
+ & + & + & + & - \\
+ & + & + & + & - \\
+ & - & - & - & - \\
+ & - & - & - & - \\
+ & - & - & - & - \\
- & - & - & - & -
\end{tabular}

$+=$ phages not found directly in either strain are generated in the mixed culture of the strains indicated in the horizontal and vertical columns.

- = no phages, other than those found directly, are generated in the mixed culture.

It follows that lysogenic properties, studied in conjunction with sensitivity to a range of phages, provide a sound biological basis for designating the new type 'Sittard', which is moreover an epidemiological entity.

Routine diagnosis must, as a matter of practicability, rely on tests of sensitivity to a set of phages rather than on the testing of lysogenic power: but the phages used for routine diagnosis should preferably be derived from tests of lysogenic power, from a study of which they derive their biological significance.

\section{Lysogenic properties of type $3 a \mathrm{I}$ (variations 1 and 2 )}

These types appeared to behave like 'Midwoud' and 'Sittard' in mixed culture with various other types. In mixed culture with strains of type 1 of Felix \& Callow they produced phages which could not be distinguished from phages found in the mixture '1 Felix \& Callow/Sittard'; but in mixed culture with strains of type 'Dundee' and also with strain 22 they produced phages which differed from those produced in mixed cultures of these types with 'Sittard'. A full report on these phages will be given elsewhere. 


\section{Other strains sensitive to the diagnostic phage for type 'Sittard'}

Preparations of the diagnostic phages from the mixed cultures 'Dundee/Sittard' have been added to the set of diagnostic phages used in the Netherlands. In addition to the strain 14487 described above, a group of strains was isolated from a small outbreak in Tilburg, which also proved to be sensitive to the 'Sittard' phage. These strains differed from type 'Beccles' in their phage reactions in that they showed discrete plaques with the test dilution of 'Beccles' phage of the Felix \& Callow system. They did not produce phage I. They were sent to Dr Felix, who found that they probably represent a new type. Their other lysogenic properties will be described elsewhere.

\section{SUMMARY AND DISCUSSION}

1. If lysogenicity is used in conjunction with sensitivity to a set of phages as a tool for phage-typing, a further subdivision of the phage types of Salm. paratyphi $B$ becomes possible, and also the types fall into a number of natural groups. When lysogenicity is tested by growth of two types in mixed culture, phages are produced which by their characteristic host ranges reveal a subdivision of types, parallel and complementary to the subdivision found by a study of lysogenicity. These phage reactions therefore possess a biological significance not entirely empirical.

2. The method of Craigie \& Yen, which made possible a great advance in phage typing of Salm. typhi by means of preparations derived by adaptation from a single phage, has not yet been shown to be applicable to the typing of Salm. paratyphi $B$ (Felix \& Callow, 1951). As some of the typing phages used in the Felix \& Callow system are identical with certain 'natural' phages derived from lysogenic strains, the lysogenic character of the types they reveal may be used in conjunction with these sensitivity tests as characteristics of phage types, if the set of phages is increased by addition of a few, shown in Table 1 . This suggestion was made to the International Committee for Enteric Phage Typing in 1952.

3. In addition to the demonstration by the direct method of phages carried by lysogenic strains of Salm. paratyphi B, phages derived from mixed cultures of different types are characteristic of some phage types. Further development of the use of such phages and study of their nature and origin demand further research. Until further investigations have been made, the subdivision of phage types which they reveal must be regarded as tentative.

4. Strains from a large outbreak of paratyphoid fever, which could not be typed reliably by the method of Felix \& Callow, possessed peculiar lysogenic properties which, together with certain phage reactions, justify their designation as a new type, 'Sittard'. These strains can be identified if phages derived from tests of lysogenic power are used in addition to those of the Felix \& Callow system. A number of strains from other sources, diagnosed as type 'Sittard' by their sensitivity to phages, were shown to possess lysogenic properties characteristic of the type.

5. A few other strains, shown in a similar way to represent new types, are described. 


\section{REFERENCES}

Boyd, J. S. K. (1950). J. Path. Bact. 62, 501.

Craigie, J. \& Brandon, K. F. (1936). J. Path. Bact. 43, 233.

Craigie, J. \& Yen, C. H. (1938). Canad. publ. Hlth. J. 29, 448, 484.

Felix, A. \& Callow, B. R. (1951). Lancet, ii, 10.

Felix, A. \& Pitt, R. M. (1934). J. Path. Bact. 38, 409.

FLo, P. C. (Personal communication.)

Hamon, Y. \& Nicolde, P. (1951). Ann. Inst. Pasteur, 80, 496.

International Committee for Enteric Phage Typing (1953). Proc. Sixth Int. Congr. Microbiol., Rome (to be published).

Lisbonne, M. \& Carrère, L. (1922). C.R. Soc. Biol., Paris, 86, 569.

Nicolle, P. \& Hamon, Y. (1951). Ann. Inst. Pasteur, 81, 614.

Nicolle, P., Hamon, Y. \& Edlinger, E. (1951). Ann. Inst. Pasteur, 80, 479.

Scholtens, R. Tн. (1936). J. Hyg., Camb., 36, 452.

Scholtens, R. TH. (1950). Leeuwenhoek ned. Tijdschr. 16, 256.

Scholtens, R. Th. (1952a). C.R. Soc. Biol., Paris, 146, 504.

Scholtens, R. Tr. (1952b). Leeuwenhoek ned. Tijdschr. 18, 257.

Sertic, V. \& Botlgakov, N. (1936). C.R. Soc. Biol., Paris, 112, 35.

WeIL, E. \& FeLIX, A. (1920). Z. ImmunForsch. 29, 24.

(MS. received for publication 26. III. 54) 